\title{
Gout nodules in vitiligo without chronic gouty arthritis: A rare presentation
}

\section{Joshi Deepak', Parasa Gayatridevi², Alluri Rajitha', Gandikota Raghurama Rao', Kolalapudi Seetharam Anjaneyulu ${ }^{1}$}

${ }^{1}$ Department of Dermatology Venereology and Leprosy, GSL Medical College, Rajahmahendravaram, AP, India, ${ }^{2}$ Department of Pathology, GSL Medical College, Rajahmahendravaram, AP, India

Corresponding author: Dr. Kolalapudi Seetharam Anjaneyulu, E-mail: Seetharamka@gmail.com

Sir,

Gouty tophi present themselves as firm and skincolored or yellowish papules or nodules with white chalky material. They may appear in any location on the body, but appear most commonly in the interphalangeal joints [1]. Clinically, they may resemble calcinosis cutis, rheumatoid nodules, xanthomas, and panniculitis. They indicate a chronic foreign-body granulomatous response to monosodium urate crystal deposits in the dermis and the subcutaneous tissue, and classically occur with untreated chronic gouty arthritis. Very rarely, in the absence of arthritis, they may be the first clinical sign of gout, which is known as gout nodulosis [2].

A 53-year-old male presented himself with multiple asymptomatic swellings over both ankles and feet persistent for four years prior. The patient had a history of depigmented patches over the face, hands, feet, and genitals since childhood, but no arthritis. His father and younger sister had vitiligo as well. The patient had been alcoholic for ten years and hypothyroid for five years, and had been taking $50 \mu \mathrm{g}$ levothyroxine sodium daily. The cutaneous examination revealed multiple skin-colored to erythematous, firm, and nontender nodules $1 \times 1 \mathrm{~cm}$ to $4 \times 5 \mathrm{~cm}$ in size, present over both lateral malleoli, the first metatarsophalangeal joint, the lateral border of the left foot, and the palmar surface of the right index finger and left thumb (Figs. la - lc). Depigmented patches were present over the face, ears, the palmar and dorsal surfaces of the hands, both feet, the scrotum, and the penis with areas of repigmentation. The complete blood count, liver function tests, serum creatinine, the fasting lipid profile, T3, T4, and TSH were normal. An abdominal ultrasound revealed left renal calculi with bilateral grade I renal parenchymal changes and grade II-III fatty changes in the liver. A radiograph of both ankles and feet showed soft tissue swellings with no evidence of erosion or joint space reduction. During a biopsy, chalky material came out from the nodule (Fig. 2). Histopathology showed compact hyperkeratosis, acanthosis, mild edema, and perivascular lymphocytic infiltrate in the upper dermis. The subcutaneous tissue was replaced by fibrillary hyaline pink material with palisading granulomas. Polarized microscopy of the chalky material proceeding from the biopsy site showed needle-shaped birefringent crystals, suggesting monosodium urate crystals (Fig. 3). This was further confirmed by a phosphotungstic acid test, in which the chalky material turned blue. Serum uric acid was high, at $8.2 \mathrm{mg} / \mathrm{dL}$ (normal range: $2.4-6 \mathrm{mg} / \mathrm{dL}$ ). Serum calcium, phosphorous, and parathormone were normal.

Gouty nodules without chronic arthritis are very rare $[3,4]$. They may occur with renal insufficiency, hyperparathyroidism, or in patients receiving antiinflammatory or diuretic drugs for a longer time. Our patient had hyperuricemia, but not renal problems, and had not received any anti-inflammatory or diuretic drugs. Also, his calcium and parathormone levels were normal. He developed gouty nodules with mild hyperuricemia, but with no arthritis and no other known cause of hyperuricemia. Kikuchi et al. reported hyperuricemia in vitiligo patients who have undergone narrowband UVB therapy. The immunomodulatory effect of UV radiation affects T lymphocytes, which attack melanocytes, resulting in increased nucleic

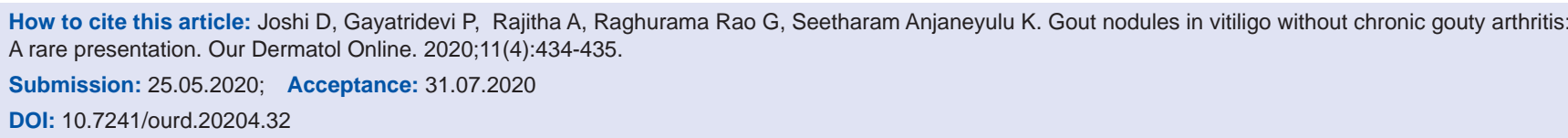



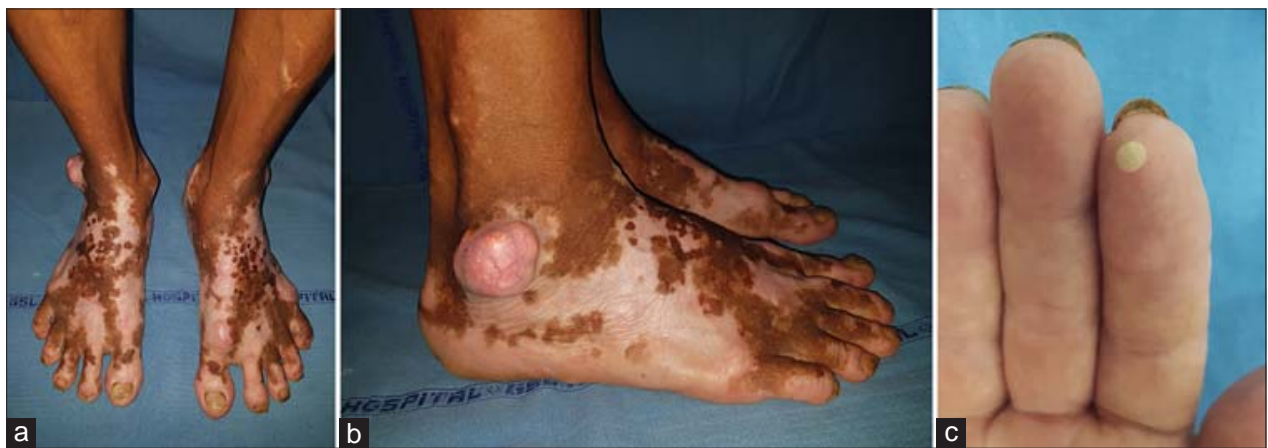

Figure 1: (a) Firm nodules over both lateral malleoli with vitiligo on both feet and ankles. (b) A nodule over the right lateral malleolus. (c) A nodule with white chalky material over the left index finger.

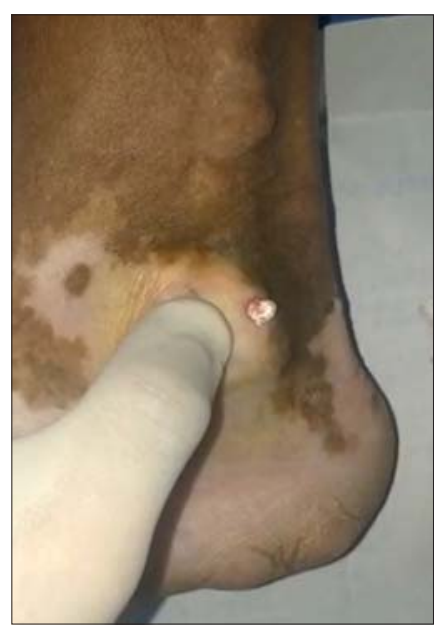

Figure 2: Chalky material from the nodule.

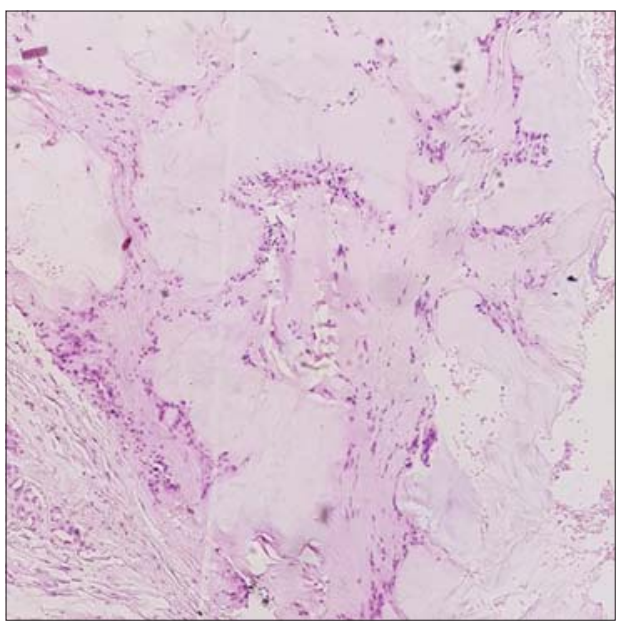

Figure 3: The subcutaneous tissue replaced by fibrillary hyaline pink material with palisading granulomas (H\&E, 400x).

acid turnover, thus causing hyperuricemia $[5,6]$. Therefore, the sun exposure in our patient, who had had vitiligo since childhood, might have been responsible for the increased uric acid levels and gouty tophi. Inflammasomes are structures that mediate the generation of IL-1. The NALP3 inflammasome is involved in gout, whereas the NALPl inflammasome in vitiligo [7]. Hence, the association of vitiligo with gout might be inflammation-mediated and induced by prolonged UV radiation.

\section{Consent}

The examination of the patient was conducted according to the principles of the Declaration of Helsinki.

The authors certify that they have obtained all appropriate patient consent forms, in which the patients gave their consent for images and other clinical information to be included in the journal. The patients understand that their names and initials will not be published and due effort will be made to conceal their identity, but that anonymity cannot be guaranteed.

\section{REFERENCES}

1. Chang P. Tophus. Our Dermatol Online. 2012;3:131-3.

2. Kumar P, Das A, Savant S, Mandal RK, Hassan S. Gout nodulosis: report of a rare case and brief review. Dermatol Online J. 2015;21:8.

3. Wernick R, Winkler C, Campbell S. Tophi as the initial manifestation of gout: report of 6 cases and review of literature. Arch Intern Med. 1992;152:873-6.

4. Chiriac A, Chirana A, Chiriac AE, Codrina A. Gout - induced by infliximab - case report. Our Dermatol Online. 2012;3:345-6.

5. Kikuchi K, Komine M, Takekoshi T, Tamaki K. Serum uric acid levels in patients with vitiligo receiving narrowband ultraviolet B phototherapy. Clin Exp Dermatol. 2007;32:107-8.

6. Rahimi BA, Farooqi K, Fazli N. Clinical patterns and associated comorbidities of vitiligo in Kandahar, Afghanistan. A case-control study. Our Dermatol Online. 2020;11:6-12.

7. Braga TT, Foresto-Neto O, Camara NOS. The role of uric acid in inflammasome-mediated kidney injury. Curr Opin Nephrol Hypertens. 2020;29:423-31.

Copyright by Joshi Deepak, et al. This is an open-access article distributed under the terms of the Creative Commons Attribution License, which permits unrestricted use, distribution, and reproduction in any medium, provided the original author and source are credited. Source of Support: Nil, Conflict of Interest: None declared. 